\title{
673.
}

\section{NOTE ON THE THEORY OF CORRESPONDENCE.}

[From the Quarterly Journal of Pure and Applied Mathematics, vol. xv. (1878), pp. 32, 33.]

IF the point $P$ on a given curve $U$ of the order $m$, and the point $Q$ on a given curve $V$ of the order $m^{\prime}$, have a $(1,1)$ correspondence, this implying that the two curves have the same deficiency; then if $P Q$ intersects the consecutive line $P^{\prime} Q^{\prime}$ in a point $R$, the locus of $R$ is a curve $W$ of the class $m+m^{\prime}$, and the point $R$ on this curve has, in general (but not universally), a $(1,1)$ correspondence with the point $P$ on $U$ or with the point $Q$ on $V$. For, considering the correspondence of the points $P$ and $R$, to a given position of $P$ there corresponds, it is clear, a single position of $R$; on the other hand, starting from $R$, the tangent at this point to the curve $W$ meets the curve $U$ in $m$ points and the curve $V$ in $m^{\prime}$ points, but it is in general only one of the $m$ points and only one of the $m^{\prime}$ points which are corresponding points on the curves $U$ and $V$; that is, it is only one of the $m$ points which is a point $P$; and the correspondence of $(P, R)$ is thus a $(1,1)$ correspondence.

But the curves $U, V$ may be such that the correspondence of $(P, R)$ is not a $(1,1)$ but a $(k, 1)$ correspondence; viz., that to a given position of $P$ there corresponds a single position of $R$, but to a given position of $R, k$ positions of $P$. To show that this is so, imagine through $P$ a line $\Pi$ having therewith a $(k, 1)$ correspondence; $P$ being, as above, a point on the curve $U$, the line in question envelopes a curve $W$; and the correspondence is such that, for any given position of $P$ on the curve $U$, we have through it a single position of the line: but, for a given tangent of the curve $W$, we have upon it $k$ positions of the point $P$, viz. $k$ of the $m$ intersections of the line with the curve $U$ are points corresponding to the line; this, of course, implies that the curve $U$ is not any curve whatever of the order $m$, but a curve of a peculiar nature. 
Imagine now that we have on the line $\Pi$ a point $Q$, having with $P$ a $(1,1)$ correspondence of a given nature: to fix the ideas, suppose $P, Q$ are harmonics in regard to a given conic: since on each of the lines $\Pi$ there are $k$ positions of $P$, there are also on the line $k$ positions of $Q$, and the locus of these $k$ points $Q$ is a curve $V$, say of the order $m^{\prime}$.

The point $P$ on the curve $U$ and the point $Q$ on the curve $V$ have a $(1,1)$ correspondence. For, consider $P$ as given: there is a single position of the line $\Pi$ intersecting $V$ in $m^{\prime}$ points, but obviously only one of these is the point $Q$. And consider $Q$ as given: then through $Q$ we have say $\mu$ tangents of the curve $W$; each of these tangents intersects the curve $U$ in $m$ points, $k$ of which are points $P$, but for a tangent taken at random no one of these is the correspondent of $Q$; it is, in general, only one of the $\mu$ tangents which has upon it $k$ points $P$, one of them being the point corresponding to $Q$; that is, to a given position of $Q$ there corresponds a single position of $P$; and the correspondence of the points $(P, Q)$ is thus a $(1,1)$ correspondence.

We have thus the point $P$ on the curve $U$ and the point $Q$ on the curve $V$, which points have with each other a $(1,1)$ correspondence; and the line $\Pi$ is the line $P Q$ joining these points; this intersects the consecutive line in a point $R$; and the locus of $R$ is the curve $W$. To a given position of $P$ there corresponds a single line $\Pi$, and therefore a single position of $R$; but to a given position of $R$ there correspond $k$ positions of $P$, viz. drawing at $R$ the tangent to the curve $W$, this is a line $\Pi$ having upon it $k$ points $P$, or the correspondence of $(P, Q)$ is, as stated, a $(k, 1)$ correspondence.

The foregoing considerations were suggested to me by the theory of parallel curves. Take a curve parallel to a given curve, for example, the ellipse; this is a curve of the order $\delta$, such that every normal thereto is a normal at two distinct points; and the curve has as its evolute the evolute of the ellipse, or, more accurately, the evolute of the ellipse taken twice; but, attending only to the evolute taken once, each tangent of the evolute is a normal of the parallel curve at two distinct points thereof, and the points of the parallel curve have with those of the evolute not a $(1,1)$ but a $(2,1)$ correspondence. 